\title{
Soft x-ray imaging with incoherent sources
}

P. Wachulak, A. Torrisi, M. Ayele, A. Bartnik, J.

Czwartos, et al. 


\title{
Soft $x$-ray imaging with incoherent sources
}

\author{
P. Wachulak ${ }^{\text {a }}$, A. Torrisi a ${ }^{\text {a }}$ M. Ayele ${ }^{\text {a }}$, A. Bartnik ${ }^{\text {a }}$, J. Czwartos a ${ }^{\text {, }}$ Ł. Węgrzyński ${ }^{\text {a }}$, \\ T. Fok ${ }^{a}$, T. Parkman ${ }^{b}, \breve{S}_{\text {. Vondrová }}{ }^{\mathrm{b}}$, J. Turňová $^{\mathrm{b}}$, M. Odstrcil ${ }^{\mathrm{c}}$ and H. Fiedorowicz ${ }^{\mathrm{a}}$ \\ ${ }^{\text {a }}$ Institute of Optoelectronics, Military University of Technology, 2, Kaliskiego Street, 00-908 \\ Warsaw, Poland \\ ${ }^{\mathrm{b}}$ Czech Technical University in Prague, Faculty of Biomedical Engineering, 27201 Kladno, Czech \\ Republic \\ ${ }^{\mathrm{c}}$ Paul Scherrer Institut, CH-5232 Villigen PSI, Switzerland
}

\begin{abstract}
In this work we present experimental, compact desk-top SXR microscope, the EUV microscope which is at this stage a technology demonstrator, and finally, the SXR contact microscope. The systems are based on laser-plasma EUV and SXR sources, employing a double stream gas puff target. The EUV and SXR full field microscopes, operating at $13.8 \mathrm{~nm}$ and $2.88 \mathrm{~nm}$ wavelengths, respectively, are capable of imaging nanostructures with a sub-50 $\mathrm{nm}$ spatial resolution with relatively short (seconds) exposure times. The SXR contact microscope operates in the "water-window" spectral range, to produce an imprint of the internal structure of the sample in a thin layer of SXR light sensitive photoresist. Applications of such desk-top EUV and SXR microscopes for studies of variety of different samples - test objects for resolution assessment and other objects such as carbon membranes, DNA plasmid samples, organic and inorganic thin layers, diatoms, algae and carcinoma cells, are also presented. Details about the sources, the microscopes as well as the imaging results for various objects will be presented and discussed. The development of such compact imaging systems may be important to the new research related to biological, material science and nanotechnology applications.
\end{abstract}

Keywords: Gas puff target, Fresnel zone plates, EUV/SXR Microscopy, Contact microscopy, Imaging, Nanometer resolution.

\section{INTRODUCTION}

Recent advancements in nanoscience and nanotechnology will surely not be possible without nanometer scale resolution imaging tools and techniques, such as extreme ultraviolet (EUV) and soft X-ray (SXR) ${ }^{1}$ microscopy. The EUV/SXR microscopy proved to be a useful tool for imaging of objects with nanometer spatial resolution and allows to obtain additional information about the objects investigated, providing at the same time high optical contrast in the specific wavelength ranges. The EUV radiation is strongly absorbed in thin layers of materials, so it is highly suitable for investigations of thin films and layers of materials, while the SXR radiation, specifically in the "water-window" $(\lambda=2.3$ - $4.4 \mathrm{~nm}$ ), is particularly suitable for high resolution biological imaging, due to high achievable contrast between carbon and water - the main constituents of biological material.

The imaging in short wavelength ranges requires a high photon flux. For this reason, until now, most of studies in this field are conducted using large scale facilities, such as synchrotron or free-electron laser installations ${ }^{2-4}$. Synchrotron and FEL facilities are being used for cutting-edge scientific experiments and provide highest available photon flux, tunability and spatial and temporal coherence. However, they also have some disadvantages (high maintenance cost, require highly trained staff due to the complexity of those sources and have limited user access). Recent progress in development of a new generation of compact EUV and SXR compact sources permits to overcome some of these limitations and allow performing experiments on much larger scale in smaller laboratories worldwide.

Laser-produced plasma sources, emitting short wavelength radiation in the SXR and EUV spectral ranges offer an important alternative to be sources dedicated for compact imaging systems, allowing to overpass the limited accessibility of large facilities and maintaining a comparable spatial resolution ${ }^{5,6}$. So far, many efforts have been made to perform nanometer spatial resolution imaging in the EUV and SXR spectral ranges over the last few years employing both large scale and compact sources. The synchrotron radiation at $\lambda=2.4 \mathrm{~nm}$ was used for imaging frozen-hydrated samples at atmospheric pressure ${ }^{7}$ to observe internal details of algae cells with a spatial resolution of $\sim 35 \mathrm{~nm}$. A SXR source, emitting at $\lambda=2.88 \mathrm{~nm}$, based on a liquid jet nitrogen target was employed recently for microscopy in the "waterwindow" range with a sub-50 $\mathrm{nm}$ spatial resolution, but this system present a very complicated hardware ${ }^{8}$.

*wachulak@gmail.com +48261 839540

X-ray Lasers and Coherent X-ray Sources: Development and Applications, edited by Annie Klisnick,

Carmen S. Menoni, Proc. of SPIE Vol. 10243, 1024300 - () 2017 SPIE

CCC code: $0277-786 \mathrm{X} / 17 / \$ 18 \cdot$ doi: $10.1117 / 12.2265093$

Proc. of SPIE Vol. 10243 1024300-1 
Another nano-imaging technique is represented by the employment of compact high order harmonic generation (HHG) sources for sub-100 nm spatial resolution ${ }^{9,10}$. However, the HHG systems require a femtosecond laser as a driver for HHG process. Also, typical HHG onversion efficiency $\left(\sim 10^{-6}-10^{-4}\right)$ often results in long exposures or does not allow a proper reconstruction of the image. Ptychographic techniques are also possible. Such techniques, usually based on employment of hard X-ray beams, although provide very high spatial resolution, are extensively time consuming ${ }^{11}$. Imaging in the EUV range permits to analyse very thin samples, nanofilms and nanostructures. This is because the EUV radiation is absorbed by solid materials with thicknesses of the order of $\sim 100 \mathrm{~nm}^{12}$ and by gaseous materials with thicknesses of the order of a few millimetres ${ }^{13}$. Employing such radiation it is also possible to visualize in a direct way through absorption contrast mechanism the flow of the gas, usually investigated with other techniques, such as the interferometry ${ }^{14}$. It was demonstrated that the radiation from a capillary discharge laser operating at a wavelength of $\lambda=$ $46.9 \mathrm{~nm}$ EUV images permits to obtain a spatial resolution better than $55 \mathrm{~nm}^{15,16}$, and that the spatial resolution of holographic images, employing the same wavelength, can be improved up to sub-50 $\mathrm{nm}^{17}$. A table top EUV laser emitting $13.2 \mathrm{~nm}$ wavelength radiation from Ni-like Cd ions was employed for imaging of with a sub-38 nm spatial resolution and a picosecond time resolution ${ }^{18}$. Using xenon gas discharge source a sub- $100 \mathrm{~nm}$ zone-plate based zooming EUV microscope was demonstrated combining Schwarzschild objective coupled to a zone plate for second stage magnification ${ }^{19}$. Very nice results were also recently demonstrated by Legall et al., ${ }^{20}$, where using liquid nitrogen target based system and $1.3 \mathrm{kHz}$ repetition rate $\mathrm{Nd}: \mathrm{YAG}$ laser they were able to record images of biological samples and nanostructures with half-pitch spatial resolution of $40-50 \mathrm{~nm}$, however, this system (laser driver especially) was quite complicated and large comparing to other compact sources. Another interesting and already well established technique for obtaining high resolution images of samples is projection imaging called contact microscopy. This method uses EUV and SXR radiation transmitted through the sample to expose a high resolution photoresist being in contact with the sample. The distance between the sample and the photoresist should be kept as small as possible to avoid blurring due to propagation and possible coherence artifacts. Using this method first imprints of human blood platelets ${ }^{21}$, fibroblasts ${ }^{22}$, diatoms ${ }^{23}$ and hydrated biological cells ${ }^{24}$ were obtained.

It is necessary, however, to find a good compromise in the desk-top SXR/EUV imaging systems, between the performance (possibility to obtain high spatial resolution and low exposure time provided by large scale facilities) and the complexity, size, cost of these setups, which is still the main limit of short wavelength photon-based microscopes. Some of these problems can be addressed employing double stream gas puff target laser plasma sources for microscopy purposes. These sources, coupled with zone plates (FZPs) optics or as illumination sources for contact microscopy, represent a very useful technique for lab-scale analysis in order to perform high spatial resolution imaging by using short wavelength radiations.

Thus, in this work we would like to show the recent developments and possible applications of three simple and compact SXR/EUV microscopes, capable of resolving 50-80 nm features that require short exposure times and have a desk-top footprint. The source employed shows different advantages, such as the possibility to generate the plasma efficiently, without debris production, they are easy to operate and offer the possibility to use continuously the EUV and SXR emission flux. Additionally, they have compact and easy construction. Moreover, the EUV/SXR microscopes, based on those sources ${ }^{25}$, do not require sample preparation such as gold coating for SEM microscopes for example. In such systems the gas puff target is produced by injection of a small amount of high- $Z$ gas - working gas, into a stream of low-Z gas - outer gas, by a fast electromagnetic double valve system, as depicted in Fig. 1a). The gas puff target is then irradiated by focused laser pulses from Nd:YAG laser, which allows generation of the EUV and SXR radiation. Both full-field microscopes use reflective optics to focus the short wavelength radiation onto a sample and FZPs to obtain magnified images of the sample with high spatial resolution, while the contact microscope uses the radiation directly, without pre-focusing, to form an imprint of the sample in the photoresist layer.

These microscopes may represent an important alternative to perform experiments in small academic laboratories and could have a huge impact speeding up nanotechnology development in the near future. The goal of developing of these microscopes is to show feasibility to achieve high resolution imaging, low exposure times, together with a compact footprint, which may, in the future, open a possibility for commercialization.

\section{DESCRIPTION OF THE MICROSCOPY SYSTEMS}

Soft X-ray contact microscope and EUV/SXR microscopes based on Fresnel optics are schematically depicted in Fig. 1b) and Fig. 1c), respectively. The sources for those microscopes were developed using a compact Nd:YAG laser, $\lambda=$ $1064 \mathrm{~nm}, 0.5-0.7 \mathrm{~J}$ pulse energy, $3 \mathrm{~ns}$ pulse duration and 1-10 $\mathrm{Hz}$ repetition rate. The laser beam is focused onto a double-stream gas puff target, schematically depicted in Fig. 1a), produced by an electromagnetic valve with double 
radially-symmetric nozzles. Laser pulses irradiate the gaseous target producing plasma. Such plasma efficiently emits radiation from various spectral ranges, including the EUV and SXR regions of electromagnetic spectrum. The gas puff target source was optimized for emissions in the "water window" (both quasi-monochromatic for the SXR full-field microscope and broadband for contact SXR microscope) and the EUV spectral ranges using spectral narrowing with thin filters, depending on the type of the microscope. A nitrogen plasma emission, filtered with Ti foil, provides $\lambda=2.88 \mathrm{~nm}$ wavelength radiation and an Ar plasma emission, filtered with $\mathrm{Mo} / \mathrm{Si}$ multilayer mirror and $\mathrm{Zr}$ filter, provides radiation at $\lambda=13.84 \mathrm{~nm}$.

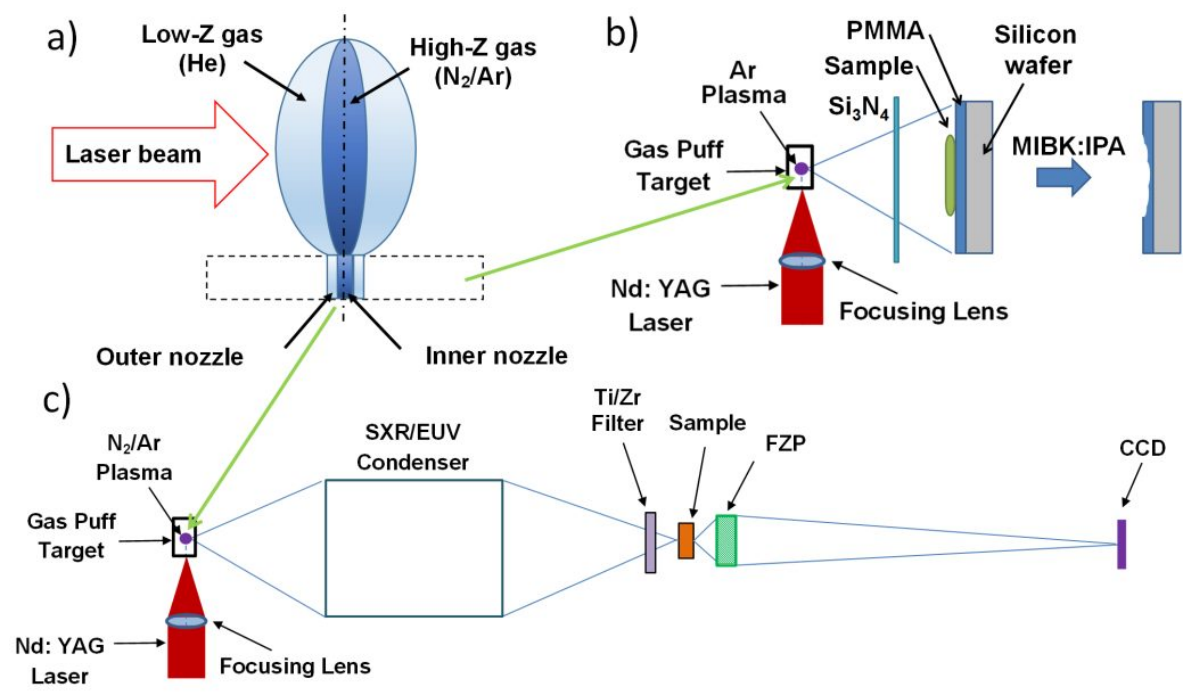

Fig. 1 - a) Scheme of the double stream gas puff target employed in laser-plasma EUV/SXR sources, b) scheme of the contact SXR microscope and c) general scheme of the SXR/EUV full-field microscopes.

However, for the argon/helium plasma if silicon nitride filter is used, it provides emission from $\lambda=2.8-4 \mathrm{~nm}$, well within the "water window" spectral range with much larger number of photons, suitable for contact microscopy. Such sources were employed for SXR and EUV experiments, respectively. The gas pressures, nozzle position in respect to the laser focus, valve timing in respect to the laser pulse were optimized in order to maximize the photon flux at the sample plane. The inner nozzle injects a small amount of working gas (high Z gas $-\mathrm{N}_{2}$ at 8 bar, in case of SXR full-field microscope, and Ar, at $10 \mathrm{bar}$, in case of the EUV full-field microscope and contact SXR microscope), while the outer nozzle injects an outer gas (low $\mathrm{Z}$ gas, He, with a pressure of 6 bar), to narrow down the flow of the working gas, reducing its density gradient along the nozzle axis. This allows obtaining higher target density at 1-2 $\mathrm{mm}$ from the nozzle exit and, in turn, allows for higher photon yield in the EUV/SXR region. Interaction of Nd:YAG laser pulses with gaseous target produces plasma, which radiation is focused using condenser optics and illuminates the sample, located in a second focal point of a condenser in the case of full-field microscopes. Depending of the type of microscope, plasma radiation is collected and focused by a different condenser and filter, and images of the objects are obtained with different zone plates using a back-illuminated CCD camera. In the case of contact SXR microscope the scheme is much simpler, because the radiation from the plasma is transmitted through a $200 \mathrm{~nm}$ thick $\mathrm{Si}_{3} \mathrm{~N}_{4}$ filter and illuminated the sample directly, to form its imprint in the surface of the photoresist. In the following paragraphs the systems will be described in more detail.

\subsection{SXR full-field microscopy system}

To focus the SXR radiation from the nitrogen plasma an ellipsoidal, axi-symmetrical SXR condenser (Rigaku, Czech Republic) coated with nickel film was used. A titanium filter (200 nm thick, Lebow), positioned downstream the condenser, selects the He-like nitrogen line at $\lambda=2.88 \mathrm{~nm}$ from nitrogen plasma emission, suitable for the objective optic. Filtered SXR radiation illuminates the sample/object, positioned $140 \mathrm{~mm}$ downstream the condenser, in the second focal point. A FZP objective (Zoneplates Ltd., UK) was used to form a magnified image onto a back-illuminated SXRsensitive CCD camera (Andor, iKon-M DO-934-BN, 1Mpixel, $13 \times 13 \mu \mathrm{m}^{2}$ pixel size) in transmission mode. A silicon nitride FZP (400 nm thick, $250 \mu \mathrm{m}$ in diameter, outer zone width of $30 \mathrm{~nm}$ ) with a focal length $\mathrm{f}=2.6 \mathrm{~mm}$ at $2.88 \mathrm{~nm}$ 
wavelength, was used as an objective. The numerical aperture of the zone plate was matched to the numerical aperture of the condenser, providing incoherent illumination ${ }^{26}$. The image plane was located $574 \mathrm{~mm}$ from the zone plate resulting in the geometrical magnification of the system of $\sim 220 x$. It was already demonstrated that such system is capable to achieve a half-pitch spatial resolution of $\sim 60 \mathrm{~nm}^{27}$. More details about the microscope and its technical component can be find in $^{28}$.

\subsection{EUV full-field microscopy system}

In this system an Ar plasma radiation was collected and spectrally narrowed by an ellipsoidal off-axis mirror coated with a Mo/Si multilayer (MLM), employed as a condenser (Reflex, Czech Republic, and IOF, Germany). The condenser was designed and manufactured in order to reflect the radiation at the wavelength of $13.5 \pm 0.5 \mathrm{~nm}$ at $45^{\circ}$ incidence angle. To eliminate longer wavelengths, emitted from the Ar plasma (at wavelengths $\lambda>16 \mathrm{~nm}$ ), a $250 \mathrm{~nm}$ thick zirconium filter (Lebow) was used. The sample was imaged using a FZP objective (diameter $200 \mu \mathrm{m}$, number of zones 1000 and outer zone width $\Delta \mathrm{r}=50 \mathrm{~nm}$, made from PMMA, from Zoneplates Ltd., UK) onto the CCD camera (Andor, iKon-M DO-934BN camera). The FZP was fabricated using e-beam lithography in a $200 \mathrm{~nm}$ thick PMMA layer deposited on top of a 50 $\mathrm{nm}$ thick $\mathrm{Si}_{3} \mathrm{~N}_{4}$ membrane. As in case of the SXR microscope, an incoherent illumination was provided by matching of the numerical aperture of the condenser and of the FZP objective. The geometrical magnification of the objective was $410 x$, however, it can be easily changed by adjusting the FZP-CCD distance. Such system, in this configuration, is capable to achieve a half-pitch spatial resolution of $48 \mathrm{~nm}^{29}$. More details about this system can be found in ${ }^{30}$.

\subsection{SXR contact microscopy system}

For this microscope the Ar plasma emission was tailored to the "water-window" spectral range by employing $200 \mathrm{~nm}$ thick $\mathrm{Si}_{3} \mathrm{~N}_{4}$ window. Using that filter the most energy will reside in the wavelength range from $2.8 \mathrm{~nm}$ to $4 \mathrm{~nm}$, well within the "water-window" range. The higher energy photons, beyond $2 \mathrm{~nm}$ and less in wavelength are not produced, due to the limitations imposed by the energy of the laser pulses and their time durations. Plasma is not sufficiently hot to emit efficiently at higher energies, while the lower energy photons will be blocked by decaying edge of the transmission curve for silicon nitride filter. Such broad band SXR radiation from Ar/He gas puff target has approximately one order of magnitude more photons than from $\mathrm{N}_{2} / \mathrm{He}$ gas puff target, which allows in this case for irradiation and exposure of the high resolution photoresist (500 $\mathrm{nm}$ thick PMMA on top of a silicon wafer). The object is then placed in contact with the PMMA. The light that is locally transmitted by the object's structure illuminates the photoresist and changes its physical and chemical structure. After the irradiation the photoresist is chemically developed in solution of methyl isobutyl ketone and isopropyl alcohol (MIBK:IPA 1:2 v/v) for 90 seconds and modulation of the light intensity absorbed by the object is converted in this process to a modulation of the thickness of the resist. This creates a relief-like structure in its surface. The height of the relief structure is directly proportional (however, not necessarily linearly), to the dose of radiation absorbed in the resist volume. In such process a high resolution imprint of the internal structure of the object can be stored in the near-surface of the photoresist and later converted to an image using for example AFM or SEM microscope. More details about this system can be found in ${ }^{31}$.

\section{RESULTS}

\subsection{SXR Full-field imaging}

Two examples of "water window" images of organic samples, acquired with the full-field SXR microscope are depicted in Fig. 2. Fig. 2a) and b) shows a sample of CT 26 fibroblast from Mus musculus colon carcinoma (strain $\mathrm{BALB} / \mathrm{c}$ ), prepared on top of a $30 \mathrm{~nm}$ thick $\mathrm{Si}_{3} \mathrm{~N}_{4}$ membrane. A direct comparison between the image acquired with a traditional optical microscope (Fig. 2a)) and the SXR microscope image (Fig. 2 b)), acquired with 200 SXR pulses, at a source repetition rate of $10 \mathrm{~Hz}$, exposure time of 22 seconds and detector $(\mathrm{CCD})$ temperature of $-20^{\circ} \mathrm{C}$, are shown. The sample was prepared with a gradual dehydration in ethanol series (final concentration $70 \% \mathrm{EtOH}$ ), without any fixation procedure. The SXR image shows improved spatial resolution due to the employment of shorter wavelength, beyond the diffraction limit of the optical-visible light microscopes. Some internal and external structures can be distinguished due to phase contrast in the visible light microscopy images and due to the modulation in the absorption of the SXR light through the sample in the SXR images. 
A second example are hippocampal neurons from E17 mouse embryos, cultured for 10 days on poly-D-lysine coated, $50 \mathrm{~nm}$ thick $\mathrm{Si}_{3} \mathrm{~N}_{4}$ substrates before fixation in $4 \% \mathrm{PFA}$ in $20 \%$ sucrose PBS followed by dehydration from $100-$ $70 \%$ ethanol and air drying. The neurons were also imaged using both microscopes: visible light microscope (40x objective, 400x magnification) - Fig. 2c) and the SXR microscope (sample to CCD magnification of 410x, exposure of 200 SXR pulses, 20 seconds) - Fig. 2d). The comparison with the optical image (Fig. 2c)) shows a significant improvement of the spatial resolution employing the "water-window" radiation. In this case it is possible to observe that the high absorption coefficient at SXR wavelengths enhances the optical contrast to the point that barely visible neuron in fig. c) is much better visualized in image d). In the SXR image it is possible to distinguish the neuron and dendrites that are blurred due to inferior resolution and phase-type imaging using the optical microscope.
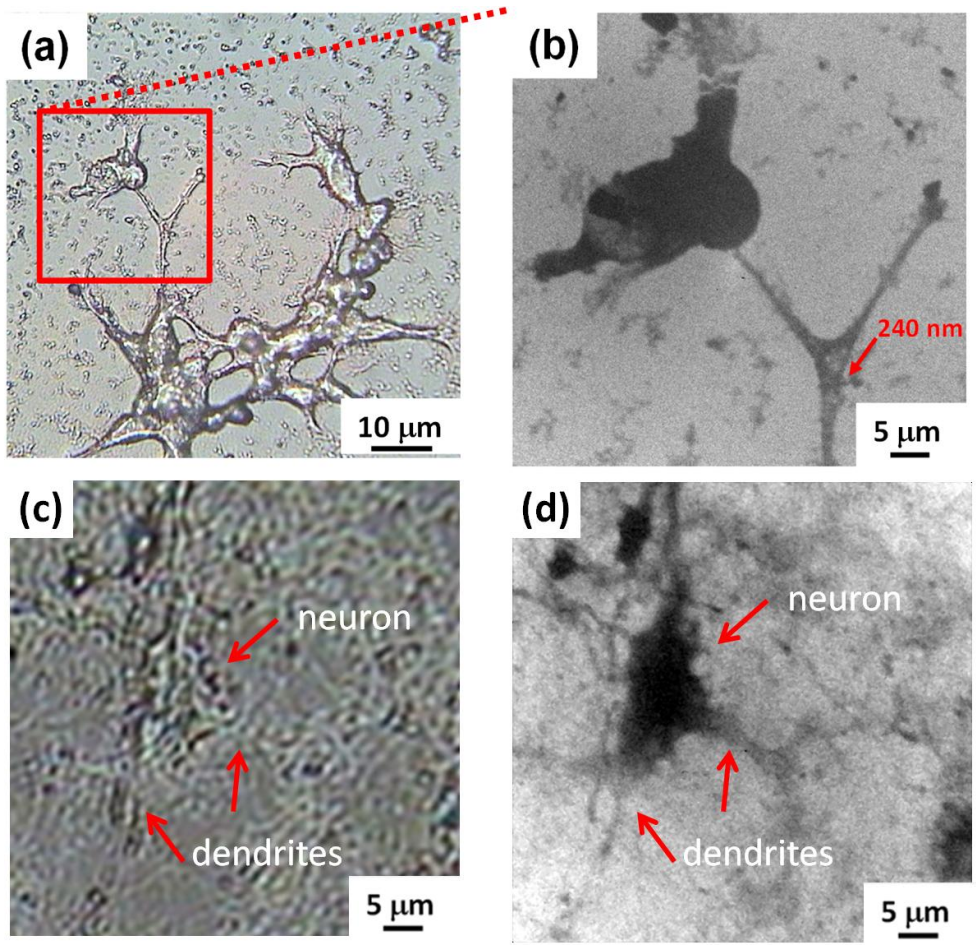

Fig. 2 - CT 26 fibroblast cells. Comparison of optical image (a) and detail imaged (b) with the SXR "water window" microscope (red square in (a)) that shows small features of the order of $240 \mathrm{~nm}$ (indicated with red arrow). Fig. (c) and (d) shows a comparison of the same mouse neurons acquired with optical microscope (40x objective, mag. 400x) and SXR microscope, respectively, showing a single neuron with dendrites branching out.

\subsection{EUV full-field imaging}

In this experiment CT26 fibroblast cells, Chrysodidimus cells and diatoms were imagined using the EUV microscope and visible light microscope. The EUV images were acquired with 200 EUV pulses - 22 sec. exposure time and detector temperature of $-20^{\circ} \mathrm{C}$. A sample of CT 26 cells fixed with $30 \%$ hexamethildisilazane (HDMS) in absolute EtOH on top of $30 \mathrm{~nm} \mathrm{Si} \mathrm{N}_{4}$ membrane was imaged with the optical microscope as depicted in Fig. 3a) and at $13.84 \mathrm{~nm}$ wavelength with the EUV microscope - Fig. 3b). It can be seen that the EUV image shows a very high contrast and resolution enhancement and permits to investigate features of the order of $100 \mathrm{~nm}$ or smaller in size.

Chrysodidimus cells imaged using the EUV microscopes can be seen Fig. 3c), while the diatoms deposited on top of $30 \mathrm{~nm}$ thick $\mathrm{Si}_{3} \mathrm{~N}_{4}$ membrane are depicted in Fig. 3d), with a small inset showing diatom structures as small as a few hundreds of nanometers. The EUV images exhibit superior spatial resolution and much higher optical magnification (410x) comparing to visible light microscopy - Fig. 3a). 

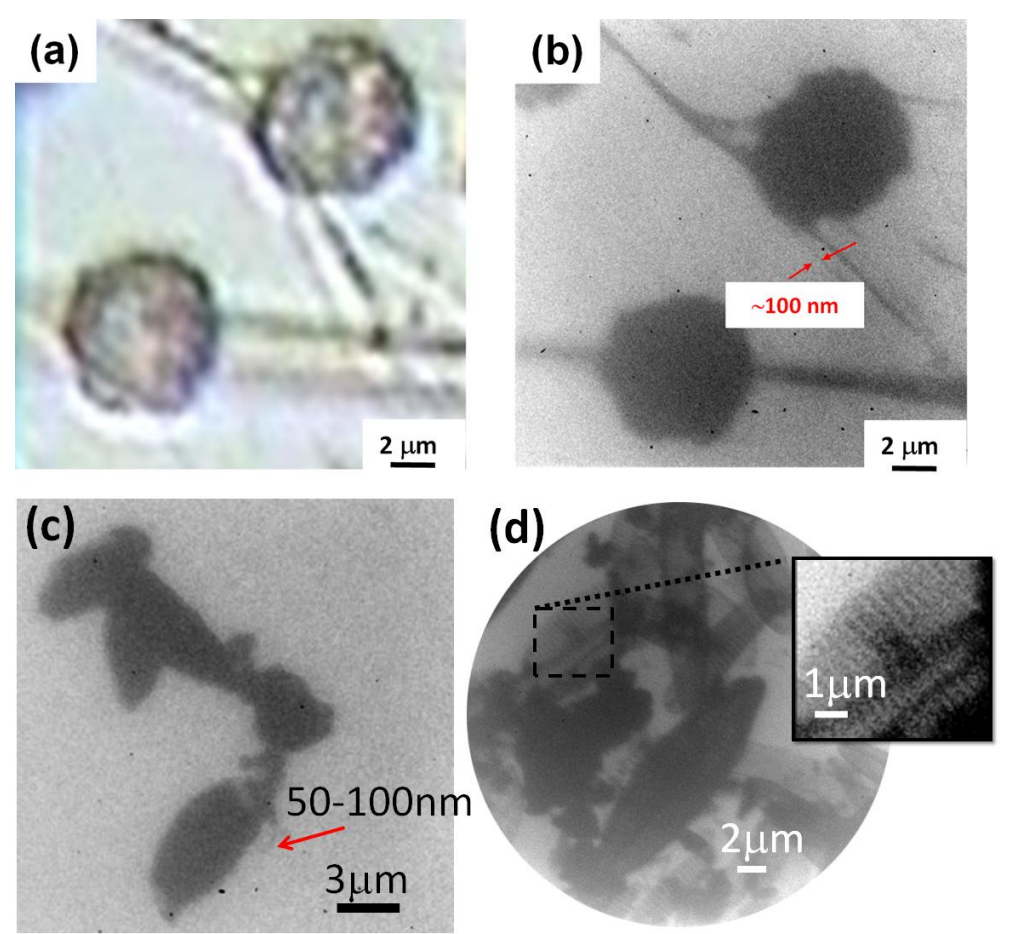

Fig. 3 - Comparison of CT 26 fibroblast cells sample acquired with optical microscope (a) and EUV microscope (b). In this last image it is possible to appreciate small features of the order of $100 \mathrm{~nm}$ (as indicated in red arrows). The EUV image of Chrysodidimus cells c) and diatoms d) obtained with exposure of 100-200 EUV pulses. Features as small as 50-100 nm are visible.

\subsection{SXR contact microscopy imaging}

The contact microscope has been used for imaging fixed epidermal cells (Keratinocytes), as depicted in Fig. 4. The sample was prepared in collaboration with the Institute of Biotechnology, Warsaw University of Technology, Poland. The sample was cultured on PMMA photoresist in a controlled culture conditions.

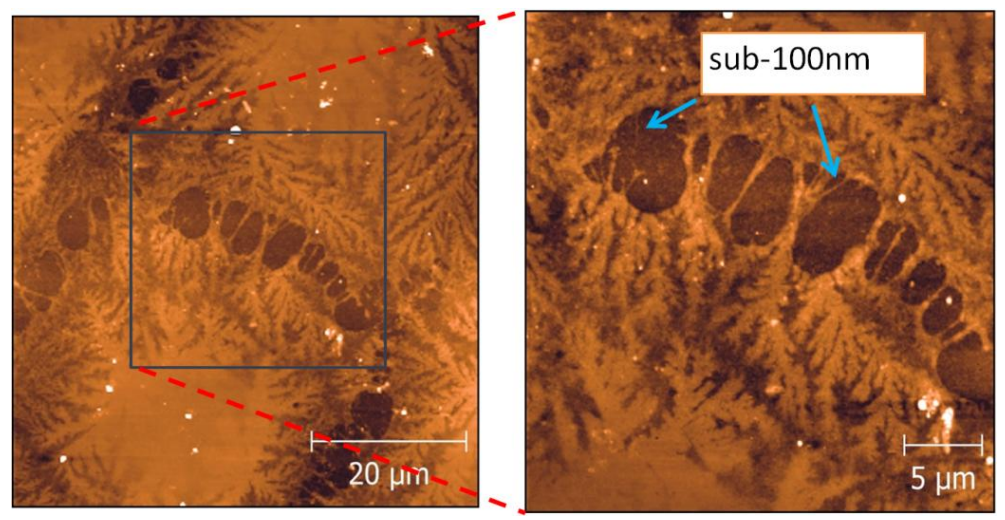

Fig. 4 - SXR contact microscopy images of fixed epidermal cells (Keratinocytes), FOV $=60 \times 60 \mu \mathrm{m}^{2}$ and $25 \mathrm{x} 25 \mu \mathrm{m}^{2}$. Images were obtained with SXR exposure of 200 pulses, 20 seconds and the photoresist was scanned using AFM microscope in semi-contact mode. Structures with sizes below $100 \mathrm{~nm}$ are visible.

A Dulbecco's Modified Eagle's Culture Medium (DMEM, Sigma-Aldrich) supplemented with 1\% L-glutamine, 1\% penicillin/streptomycin and 10\% heat inactivated foetal bovine serum (Celbio) was used as a culture medium. The culture medium was washed with PBS buffer solution followed by fixation and dehydration of the cells using paraformaldehyde $3.7 \%$ in phosphate buffered saline at $35^{\circ} \mathrm{C}$. 
The exposure of 200 SXR pulses, 20 second exposure time, was required to expose the PMMA photoresist and reach spatial resolution of approximately $80 \mathrm{~nm}$ half-pitch. The photoresist was treated with previously mentioned procedure and later scanned with AFM (AFM, NT-MTD, Russia) operating in semi-contact mode. Each image was 512x512 points over $60 \times 60 \mu \mathrm{m}^{2}$ and $25 \times 25 \mu \mathrm{m}^{2}$ in left and right images, respectively. As can be observed, structures with sizes below $100 \mathrm{~nm}$ are clearly visible. More details about this work can be found in ${ }^{31}$.

\section{SUMMARY AND CONCLUSIONS}

The SXR/EUV desk-top microscopes, both full-field and contact-type, based on laser plasma sources with double stream gas puff target allow capturing magnified images of the samples, with 50-80 nm half-pitch spatial resolution and exposure time as low as few seconds. The microscopes are compact and permit to obtain images with a nanometer spatial resolution and short exposure time. Such characteristics represent key parameters which open possibility of a future commercialization of such systems. Those systems employ photons and not electrons, like in SEM, allow obtaining additional/complementary information about the sample. Their spatial resolution is comparable to what is possible to obtain with Stimulated Emission Depletion microscopy (STED) $)^{32,33}$, however, such table-top systems allow for a direct acquisition of a full field images, in contrary to STED acquisition, operating in scanning mode and do not require fluorescent bio markers or staining that can modify the morphology of the sample. Additionally, they use wavelengths providing high optical contrast, either in biological (SXR microscope) or in all matter (EUV microscope).

\section{ACKNOWLEDGEMENTS}

This work is supported by the National Science Centre, Opus programme, grant agreement number UMO2015/17/B/ST7/03718 and UMO-2015/19/B/ST3/00435, the Education, Audiovisual and Culture Executive Agency (EACEA) Erasmus Mundus Joint Doctorate Programme Project No. 2012-0033 and from the European Union's Horizon 2020 research and innovation program, under Laserlab-Europe IV, grant agreement No. 654148. We would like to thank to Dr. Andrzej Kowalik and Mrs. Anna Rojek, from Institute of Electronic Materials Technology (ITME), Warsaw, Poland for the preparation of the photoresist in their laboratory. We also acknowledge Dr. Tomasz Kobiela and Mrs. Anna Sobiepanek, from the Faculty of Chemistry Warsaw University of Technology for providing biological samples used for contact microscopy experiments.

\section{REFERENCES}

[1] The International Organization for Standardization., "ISO 21348 Definitions of Solar Irradiance Spectral Categories," 6-7 (2007).

[2] Gros, M. A. Le., Mcdermott, G.., Cinquin, B. P., "Biological soft X-ray tomography on beamline 2 . 1 at the Advanced Light Source," Journal of Synchrotron Radiation 21 (6), 1-8 (2014).

[3] Chapman, H. N., Barty, A., Bogan, M. J., Ebastien, S., Frank, M., Hau-riege, S. P., Marchesini, S., Woods, B. W., Sa, S. A., et al., "Femtosecond diffractive imaging with a soft-X-ray free-electron laser," Nature Physics 2, 839-843 (2006).

[4] Wilke, R. N., Priebe, M., Bartels, M., Giewekemeyer, K., Diaz, A., Karvinen, P., Salditt, T., "Hard X-ray imaging of bacterial cells: nano-diffraction and ptychographic reconstruction," Optics Express 20 (17), 19232 (2012).

[5] Marino, S., Palanco, S., Gabás, M., Romero, R.., Ramos-Barrado, J. R., "Laser nano- and micro-structuring of silicon using a laser-induced plasma for beam conditioning," Nanotechnology 26 (5), 55303, IOP Publishing (2015).

[6] Van Malderen, S. J. M., van Elteren, J. T., Vanhaecke, F., "Submicrometer Imaging by Laser AblationInductively Coupled Plasma Mass Spectrometry via Signal and Image Deconvolution Approaches," Analytical chemistry 87 (12), 6125-6132 (2015).

[7] Schneider, G., "Cryo X-ray microscopy with high spatial resolution in amplitude and phase contrast," Ultramicroscopy 75, 85-104 (1998).

[8] Kim, K. W., Kwon, Y., Nam, K., Lim, J., Kim, K. W., Chon, K., Kim, B., Kim, D. E., Kim, J., et al., “Compact soft x-ray transmission microscopy with sub-50 nm spatial resolution," Physics in Medicine and Biology 51 (6), N99-N107 (2006). 
[9] Kim, H. T., Kim, I. J., Kim, C. M., Yu, T. J., Lee, S. K., Sung, J. H., Yoon, J. W., Yun, H., Jeong, T. M., et al., "Single-Shot Nanometer-Scale Fourier Transform Hologram Using Ni-like Ag X-Ray Laser," Springer Proceedings in Physics, 323-328 (2010).

[10] Park, J. J. J., Kim, D. S., Jeon, S. C., Park, J. J. J., Lee, K. H., Lee, J., Kim, K. N., Yoo, J. J.., Nam, C. H., "Table-top soft x-ray microscope adopting a PMMA phase-reversal zone plate," Optics Letters 2 (3), 5-6 (2009).

[11] Dierolf, M., Thibault, P., Menzel, A., Kewish, C. M., Jefimovs, K., Schlichting, U., Von König, K., Bunk, O., Pfeiffer, F., "Ptychographic coherent diffractive imaging of weakly scattering specimens," New Journal of Physics 12, 035017 (2010).

[12] Marconi, M. C., Wachulak, P. W., "Extreme ultraviolet lithography with table top lasers," Progress in Quantum Electronics 34 (4), 173-190 (2010).

[13] Wachulak, P. W., Wegrzynski, L., Bartnik, A., Fok, T., Jarocki, R., Kostecki, J., Szczurek, M.., Fiedorowicz, H., "Characterization of a dual-gas multi-jet gas puff target for high-order harmonic generation using extreme ultraviolet shadowgraphy," Laser and Particle Beams 31(2), 219-227 (2013).

[14] Li, T., Li, G., Wu, L., Yuan, L., Yu, B., Wang, X., Yang, H., Wu, L., Wang, L., et al., "Analysis and design of imaging Fabry-Perot interferometers for measurement of Rayleigh-Brillouin scattering spectra in gas flows," SPIE Proceeding 8910, 89101E (2013).

[15] Brewer, C., Brizuela, F., Wachulak, P., Martz, D. H., Chao, W., Anderson, E. H., Attwood, D. T., Vinogradov, A. V., Artyukov, I. a., et al., "Single-shot extreme ultraviolet laser imaging of nanostructures with wavelength resolution," Optics letters 33 (5), 518-520 (2008).

[16] Wachulak, P. W., Brewer, C. A., Brizuela, F., Menoni, C. S., Chao, W., Anderson, E. H., Bartels, R. A., Rocca, J. J.,, Marconi, M. C., "Analysis of extreme ultraviolet microscopy images of patterned nanostructures based on a correlation method," Journal of the Optical Society of America B 25 (7), B20 (2008).

[17] Wachulak, P. W., Marconi, M. C., Bartels, R. a., Menoni, C. S.., Rocca, J. J., "Soft x-ray laser holography with wavelength resolution," Journal of the Optical Society of America B 25 (11), 1811-1814 (2008).

[18] Vaschenko, G., Brizuela, F., Brewer, C., Grisham, M., Mancini, H., Menoni, C. S., Marconi, M. C., Rocca, J. J., Chao, W., et al., "Nanoimaging with a compact extreme-ultraviolet laser," Optics letters 30 (16), 2095-2097 (2005).

[19] Juschkin, L., Freiberger, R.., Bergmann, K., "EUV microscopy for defect inspection by dark-field mapping and zone plate zooming," Journal of Physics: Conference Series 186, 12030 (2009).

[20] Legall, H., Blobel, G., Stiel, H., Sandner, W., Seim, C., Takman, P., Martz, D. H., Selin, M., Vogt, U., “Compact x-ray microscope for the water window based on a high brightness laser plasma source," Optics Express 20 (16), 18362-18369 (2012).

[21] Kirz, J., Jacobsen, C., Howells, M., "Soft X-ray microscopes and their biological applications.," Quarterly reviews of biophysics 28 (1), 33-130 (1995).

[22] Cheng, P. C., Feder, R., Shinozaki, D. M., Tan, K. H., Eason, R. W., Michette, A., Rosser, R. J., "Soft X-ray contact microscopy," Nuclear Instruments and Methods in Physics Research A 246, 668-674 (1986).

[23] Ford, T. W., Stead, A. D., Cotton, R. A., "Soft X-ray contact microscopy of biological materials," Electron Microscopy Reviews 4 (2), 269-292 (1991).

[24] Kado, M., Kishimoto, M., Tamotsu, S., Yasuda, K., Aoyama, M., Shinohara, K., "Imaging of fine structures of cellular organelles in hydrated biological cells by a soft $\mathrm{x}$-ray microscope combined with a fluorescence microscope," X-Ray Lasers and Coherent X-Ray Sources: Development and Applications X 8849, 1-7 (2013).

[25] Wachulak, P. W., Bartnik, A., Skorupka, M., Kostecki, J., Jarocki, R., Szczurek, M. M., Wegrzynski, L., Fok, T., Fiedorowicz, H., "Water-window microscopy using a compact, laser-plasma SXR source based on a doublestream gas-puff target," Applied Physics B: Lasers and Optics 111 (2), 239-247 (2013).

[26] Heck, J. M., Attwood, D. T., Berkeley, E. O. L., Meyer-ilse, W., Anderson, E. H., Berkeley, E. O. L., "Resolution determination in X-ray microscopy: an analysis of the effects of partial coherence and illumination spectrum," Journal of X-Ray Science and Technology 8, 95-104 (1998).

[27] Wachulak, P., Torrisi, A., Nawaz, M. F., Bartnik, A., Adjei, D., Vondrová, Š., Turňová, J., Jančarek, A., Limpouch, J., et al., "A Compact 'Water Window' Microscope with $60 \mathrm{~nm}$ Spatial Resolution for Applications in Biology and Nanotechnology," Microscopy and Microanalysis 21 (5), 1214-1223 (2015).

[28] Wachulak, P. W., Torrisi, A., Bartnik, A., Adjei, D., Kostecki, J., Wegrzynski, L., Jarocki, R., Szczurek, M.., Fiedorowicz, H., "Desktop water window microscope using a double-stream gas puff target source," Applied Physics B 118 (4), 573-578 (2015).

[29] Wachulak, P. W., Torrisi, A., Bartnik, A., Węgrzyński, Ł., Fok, T.., Fiedorowicz, H., "A desk-top extreme ultraviolet microscope based on a compact laser-plasma light source," Applied Physics B 123 (25), 1-5 (2016). 
[30] Torrisi, A., Wachulak, P., Węgrzyński, Ł., Fok, T., Bartnik, A., Parkman, T., Vondrová, Š., Turňová, J., Jankiewicz, B. J., et al., "A stand-alone compact EUV microscope based on gas-puff target sourcele," Journal of Microscopy 265 (2), 251-260 (2017).

[31] Ayele, M. G., Czwartos, J., Adjei, D., Wachulak, P., Ahad, I. U., Bartnik, A., Wegrzynski, Ł., Szczurek, M., Jarocki, R., et al., "Contact Microscopy using a Compact Laser Produced Plasma Soft X-Ray Source," Acta Physica Polonica A 129 (2), 237-240 (2016).

[32] Otomo, K., Hibi, T., Kozawa, Y., "STED microscopy - super-resolution bio-imaging utilizing a stimulated emission depletion," Microscopy 64 (4), 1-10 (2015).

[33] Farahani, J. N., Schibler, M. J.., Bentolila, L. A., "Stimulated Emission Depletion (STED) Microscopy: from Theory to Practice," Microscopy: Science, Technology, Applications and Education 2 (4), 1539-1547 (2010). 